\title{
Taking the pain away
}

\author{
Cholawat Pacharinsak, DVM, MS, PhD, DACVAA, Assistant Professor, \\ Director of Anesthesia, Pain Management, and Surgery, Department of \\ Comparative Medicine, Stanford University School of Medicine, Stanford, CA
}

Dr. Pacharinsak talks about splitting time between his role as a laboratory animal anesthesiologist and his research on improving pain management for research animals.

\begin{abstract}
How did you become interested in anesthesiology and pain management?

I knew that I wanted to become a veterinary anesthesiologist when I was a clinician in Thailand, where anesthesiologists were scarce in both veterinary practice and veterinary schools. Anesthesia and pain management does not end when the animals wake up; it continues into the intensive care unit and the post-operative care period. I enjoy the challenge of understanding what is physiologically going on with an animal by monitoring many aspects of the animal's health and having to think or act fast in reaction to unexpected events.
\end{abstract}

\section{Your research focuses on understanding the neurobiology of cancer pain and improving clinical pain management. Tell us about why this work is so important to you.}

I have had an interest in pain management since starting my anesthesiology residency training at Washington State University. I am fortunate to have had great mentors who encouraged me to further study the neurobiology of pain during my $\mathrm{PhD}$ work at the University of Minnesota. In the last decade, significant progress has been made in the understanding of pain pathways, but what we know today is just a fraction of what is actually happening. Although pain is part of the body's normal protective process against further injury, when it goes untreated or becomes chronic, it is difficult to alleviate. Although peri- and post-operative pain is easier to control than chronic pain, I am focusing on finding more effective ways to alleviate pain in research animals, whether through drug discovery, effective drug administration techniques or improved pain evaluation methods. I believe that with our current knowledge, there should be better ways to detect pain and provide analgesia to animals.

I also study cancer pain. With advanced technologies and treatments, cancer patients can live longer, but they still have to experience the pain induced by cancer. Because cancer pain is chronic and usually difficult to treat, it responds poorly to conventional analgesics. An improved understanding of how cancer induces pain will lead to effective treatments that improve the quality of life for both animals and humans.

\section{How do you manage to balance your clinical work with your research activities?}

I have to say it is a time management challenge. Investigators and surgeons have to schedule their cases on the calendar ahead of time, so I balance my research accordingly. When I don't have cases to supervise or perform myself, I write grant applications and reports and other publications, do my own research, teach classes and workshops or prepare conference presentations. Whenever possible, I try to collaborate with researchers who can benefit from my specialty so that I am providing a service as well as gaining publishable results. The great thing is that Stanford University completely supports

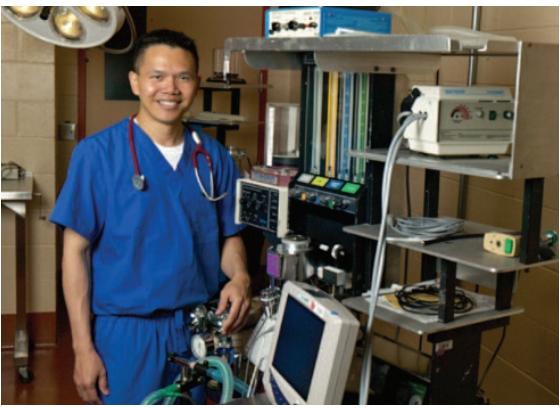

faculty research, and this encourages me to seek out these opportunities.

\section{What do you enjoy most about your career?}

I really enjoy being able to apply my knowledge in anesthesia, intensive care and pain management situations that benefit research animals both during and after various procedures. For instance, during anesthesia, I perform intensive monitoring which lets me detect when things are not going as expected (e.g., blood loss, hypotension, hypothermia, arrhythmia, etc.).

Being in an academic environment, nothing has satisfied me more than teaching, interacting with and mentoring students. In addition, I enjoy working with experts in veterinary medicine, human medicine and other specialties like bioengineering and anthropology. I generally work closely with investigators, and I can't emphasize enough that being a team player is an important part of animal welfare: good workplace relationships are crucial to successful case outcomes. Many times, I have received gratitude from researchers for my role. It is nice knowing that I contribute to research that has a significant and positive impact on animal and human health. My ability to help others and provide for animal welfare gives me great job satisfaction.

This is how I see things: You don't have to be a straight-A student to be successful. You have to follow your passion: do what you love and love what you do. I love what I do, and I'm lucky that I'm able to do what I love. 